\title{
Planning for retirement from medicine: a mixed-methods study
}

\author{
Michelle Pannor Silver PhD, Laura K. Easty MDCM
}

Abstract

Background: Evidence suggests there are important personal and social consequences associated with inadequate retirement planning for physicians. We evaluated whether academic physicians felt satisfied with their retirement planning, and identified obstacles to retirement planning and a set of factors to facilitate retirement planning.

Methods: We applied a sequential mixed-methods research design to explore and examine factors that facilitate academic physician retirement planning using data collected from multiple sources (including 7 focus groups, an internet-based survey and 23 in-depth interviews). We examined survey results regarding retirement planning satisfaction and preferences for complete versus gradual retirement. We used thematic analysis to examine verbatim transcripts and notes from the focus groups and interviews.

Results: Survey data (response rate $51 \%$ ) indicated that $10 \%$ of respondents were very satisfied with their retirement planning and $89.5 \%$ would prefer to retire gradually rather than stop work completely. Key barriers to retirement planning that emerged included poor personal financial management, rigid institutional structures and professional norms. Facilitators included financial planning resources for physicians at multiple career stages, opportunities and resources for later-career transitions and later-career mentorship support for intergenerational collaboration, and recognition of retirees.

Interpretation: Key findings highlight perceived barriers to retirement planning at various career stages in addition to factors that can enhance physicians' retirement planning, including creating gradual and flexible retirement options, supporting ongoing discussions about financial planning and later career transitions, and fostering a culture that continues to honour and involve retirees. Medical institutions could foster innovative models for later-career transitions from medicine in ways that address physicians' needs at various career stages, support gradual transitions from practice and recognize the value of experienced, capable later-career physicians and retirees.

$\mathrm{P}$ hysicians face particular challenges in planning for later-career transitions, as evidenced by trends toward both early burnout and later retirement. ${ }^{1-4}$ Unfortunately, physicians tend to struggle with financial management throughout their careers, ${ }^{5,6}$ with limited personal time, and thus may be unable, too busy, unwilling or even unaware of the need to plan for their retirement. ${ }^{7,8}$ These challenges are exacerbated by the likelihood that Canadian physicians will not have an employer-sponsored pension and must take the initiative to invest for retirement independently. ${ }^{9,10}$

Inadequate retirement planning can have consequences for both patient care and the individual physician. For the individual, retiring without sufficient planning risks an abrupt loss of their professional social network and threatens the identity and purpose that their career provides. ${ }^{711,12}$ Physician retirement, without transition planning for suitable replacements, can lead to shortages and raise concerns about patient continuity of care. ${ }^{13-15}$ Although there are numerous benefits to patients that come from having an experienced physician, ${ }^{16}$ concerns have been raised with regard to the personal health burdens experienced by older physicians ${ }^{17}$ and for patient safety, $7,18,19$ particularly in procedural specialties such as surgery. ${ }^{20}$ Therefore, successful physician retirement requires planning for the financial demands, physical changes and psychosocial dynamics that are associated with aging and later life transitions. ${ }^{21-23}$ Some strategies for approaching physician retirement have already been discussed, including full mandatory retirement based on age, ${ }^{24}$ phased retirement plans in some American academic institution $s^{25}$ and a preference for

Competing interests: None declared.

Correspondence to: Michelle Pannor Silver, michelle.silver@ utoronto.ca CMAJ Open 2017. DOI:10.9778/cmajo.20160133 
part-time work among pediatricians transitioning to retirement. ${ }^{26}$ However, less is known about the extent to which physicians are satisfied with their own retirement planning, their preferences regarding gradual versus full retirement, perceived barriers to retirement and factors that facilitate retirement planning.

The objective of this study was to contribute to a better understanding of factors that facilitate physician retirement planning. We decided to focus on the 4 aforementioned aspects of retirement planning by examining the perspectives of physicians from a range of different career stages working in an academic medical setting. Academic medicine presents a unique vantage point because the stakes of succession planning tend to be high given the difficulty associated with finding suitable replacements for a retiree. ${ }^{27}$ In addition, the strong work identity ${ }^{11,28}$ and affiliation of academic physicians with multiple institutions through practice, research and teaching results in work-life balance challenges ${ }^{29}$ that leave little time for planning and add a complexity to retirement planning that makes for an ideal point of reference. Finally, because academic centres are the vehicle through which most physicians, regardless of their ultimate practice location, receive their training, academic medicine plays a substantial role in shaping physician norms.

\section{Methods}

\section{Design}

We used a sequential mixed-methods research design ${ }^{30}$ to explore and examine factors that facilitate academic physician retirement planning in consultation with members of the University of Toronto Department of Medicine Faculty Development Committee. The use of mixed methods is gaining momentum in medicine ${ }^{31,32}$ and gerontology, ${ }^{33}$ which suggests that combining qualitative and quantitative approaches in a single study can prove to be a helpful way of providing a more holistic understanding of contemporary phenomena. ${ }^{34}$ The study design (Figure 1) included an initial exploratory stage that featured focus groups that were examined and used to inform the subsequent online survey questions. These steps were followed by interviews conducted by the lead author (M.P.S.). The design and data collection for this study were developed by the lead author in collaboration with the Department of Medicine Faculty Development Committee. The lead author is not a physician and has no personal relationships with participants in this study. The coauthor is a physician who worked with the lead author as a graduate stu- dent. The coauthor was not involved in data collection and had no direct contact with participants, but assisted with the analysis and integration of anonymized data.

\section{Setting}

The University of Toronto Department of Medicine is one of the oldest and largest departments of medicine in North America, with 19 subspecialty divisions across multiple sites. This setting allowed us to collect data from an urban sample of practising academic physicians working at 11 different hospitals and all 19 medical specialties. About 800 full-time faculty members were eligible for participation.

\section{Data collection}

Data collection was designed and administered through a collaborative process, between the lead author and members of the University of Toronto Department of Medicine Faculty Development Committee. The survey was administered directly by the Department of Medicine, and all qualitative components of the study were conducted by the lead author. Names and any information that might compromise the confidentiality of participants or qualitative components of this study are not reported to preserve confidentiality in accordance with all informed consent protocols.

\section{Focus groups}

Focus groups were chosen as the primary data collection method for the preliminary stage of the study to support open-ended answers and participant-led thematic discussions. The lead author coordinated 7 focus groups between June and September 2014 to obtain a preliminary understanding of academic physicians' perceptions about retirement. Eligible participants were recruited by a member of the administrative staff from the department of medicine through email. All eligible participants were provided with a letter of information about the study. When an eligible participant indicated his or her availability and interest in the study, he or she was sent additional information about the study.

Stratified purposive sampling was employed to ensure inclusion of sex, years of work experience, rank (assistant professors v. associate professors, and full professors) and academic stream (educator, clinician, researcher and administrator). Three focus groups were conducted with academic physicians in early career stages, defined as having fewer than 10 years of full-time work experience in academic medicine and 4 focus groups were held with academic physicians who had 10 years or more of full-time work experience in aca-

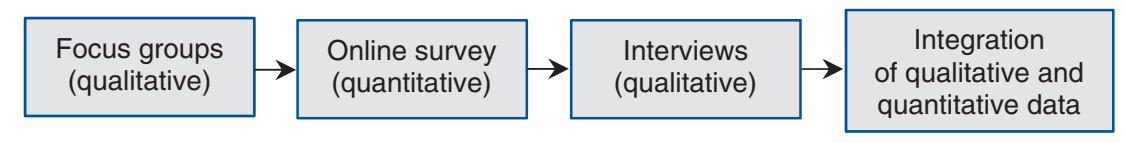

Figure 1: Sequential mixed-methods design. 
demic medicine and who were more than 50 years of age. Each focus group included 3 or 4 participants and was led by 1 facilitator. Participants in the focus groups were asked a series of questions about their perceptions of retirement (see Appendix 1, available at www.cmajopen.ca/content/5/1/E123/ suppl/DC1. All focus groups were audio recorded and transcribed verbatim. Each focus group lasted about 60 minutes. Field notes were made during and after each focus group. Validation of verbatim transcription was conducted for data quality assurance. Identifying participant information was removed by the lead author before analysis.

\section{Online survey}

The online survey was distributed to all active members of the University of Toronto Department of Medicine. The survey was posted on a website, and responses were collected in the winter of 2015. The lead author designed a set of survey questions that were included as a module on retirement and embedded in the department's larger biannual survey after they were edited and approved by the Faculty Development Committee. Survey questions reported here focus on participants' retirement plans and degree of satisfaction with their retirement planning (see Appendix 2, available at www.cmajopen.ca/content/5/1/E123/suppl/DC1).

\section{Interviews}

The lead author conducted in-depth semistructured interviews with academic physicians in administrative leadership positions. For the interviews, a stratified purposive sampling method was used to ensure inclusion of sex, clinical specialty and hospital affiliation. Participants in the interviews were asked a series of questions about the extent to which they had planned for their retirement, and factors they perceived as barriers to and facilitators of physician retirement planning (see Appendix 3 available at www.cmajopen.ca/content/5/1/ E123/suppl/DC1. The interview guide was pilot tested, prompts were provided by the interviewer, and repeat interviews were not carried out. Each interview lasted about 60 minutes. Field notes were made during and after each interview. All interviews were audio recorded and transcribed verbatim. Validation of verbatim transcription was conducted for data quality assurance. Identifying participant information was removed by the lead author before analysis.

\section{Analysis}

Transcripts from the focus groups were read several times by each author. The authors met multiple times to discuss, contrast and deliberate the themes that emerged using thematic analysis. $^{35,36}$ The survey results were examined to adjust for differences in responses to the questions about retirement planning satisfaction and preferences for complete versus gradual retirement based on respondents' years of experience and sex using Pearson $\chi^{2}$ tests. Transcripts from the interviews were examined by the lead author to further develop the emergent themes related to barriers and facilitators of retirement planning. In addition, member checking ${ }^{37,38}$ was performed to help confirm authors' interpretations with quoted participants by presenting summary thematic analyses to 11 participants selected on the basis of their work as an academic physician at 1 of each of the 11 hospitals affiliated with the department. After separate analyses of each component of this study, data were integrated with the intent of providing mutual illumination of findings and a richer understanding of factors that facilitate physician retirement planning.

\section{Ethics approval}

This study was approved by the University of Toronto Research Ethics Board.

\section{Resullts}

Characteristics of focus group participants are reported in Table 1 . The online survey was sent to 362 people, and had a $51 \%$ response rate. Characteristics of the survey participants are reported in Table 2 . All participants who initiated the survey completed it.

Characteristics of interviewees $(n=26)$ are not reported to preserve anonymity. Requests for participation were sent out via email through the 19 subspeciality divisions, and only eligible full-time faculty who read the information about the study and agreed to participate were included in the focus groups and interviews. Interviews were conducted until the point that little new information was expected to be learned from further interviews.

Online survey results indicated that respondents would prefer to retire gradually $(89.5 \%)$ rather than completely stop

Table 1: Characteristics of focus group participants $(n=25)$

\begin{tabular}{|c|c|}
\hline Characteristic & No. (\%) \\
\hline \multicolumn{2}{|l|}{ Sex } \\
\hline Male & $15(60.0)$ \\
\hline \multicolumn{2}{|l|}{ Career stage } \\
\hline Early* & $9(36.0)$ \\
\hline Later† & $16(64.0)$ \\
\hline \multicolumn{2}{|l|}{ Rank } \\
\hline Assistant professor & $12(48.0)$ \\
\hline Associate professor & $6(24.0)$ \\
\hline Full professor & $7(28.0)$ \\
\hline \multicolumn{2}{|l|}{ Job description } \\
\hline Administrator & $5(20.0)$ \\
\hline Educator/teacher & $5(20.0)$ \\
\hline Investigator & $7(28.0)$ \\
\hline Scientist & $8(32.0)$ \\
\hline \multicolumn{2}{|c|}{$\begin{array}{l}{ }^{*} \text { Early career participants were defined as having fewer than } 10 \text { years of full-time } \\
\text { work experience in academic medicine. } \\
\text { †Later career participants were defined as having } 10 \text { years or more of full-time } \\
\text { work experience in academic medicine and being more than } 50 \text { years of age. }\end{array}$} \\
\hline
\end{tabular}




\begin{tabular}{|lc|}
\hline \multicolumn{2}{|l|}{ Table 2: Characteristics of survey respondents $(\boldsymbol{n}=\mathbf{3 6 2})$} \\
\hline Characteristic & No. $(\%)$ \\
\hline Sex & $218(60.2)$ \\
\hline Male & \\
\hline Years of experience & $127(35.1)$ \\
\hline $0-8$ & $114(31.5)$ \\
\hline $9-17$ & $121(33.4)$ \\
\hline$\geq 18$ & $10(2.8)$ \\
\hline Job description & $158(43.6)$ \\
\hline Administrator & $76(21.0)$ \\
\hline Educator/teacher & $102(28.1)$ \\
\hline Investigator & $16(4.4)$ \\
\hline Scientist & \\
\hline Quality innovation & \\
\hline
\end{tabular}

working, and only $10 \%$ of respondents were very satisfied with their retirement planning. There were no significant differences by years of experience or sex with regard to preferences for complete versus gradual retirement. Findings from interviews reinforced key barriers to retirement planning that could explain low satisfaction and specific concerns about retiring gradually. Themes that emerged from analysis of the focus group discussions and interviews provided richer descriptive accounts of barriers and facilitators to retirement planning as described below and shown through quotes in Table 3.

\section{Barriers to retirement planning}

\section{Poor personal financial management}

Most participants acknowledged that they did not have a clear sense of their own financial situation as it related to retirement. Both early and later-career focus group participants lacked a clear vision or trust in their own long-term financial plans, even after mentioning that they had a financial advisor, a living will or a health care power of attorney. Participants described their situation as akin to being on a treadmill; working more meant greater earnings, which created an incentive to focus on the present and a disincentive in terms of planning for retirement.

Several participants discussed how their complex lives presented barriers to planning for retirement. For example, having trained or worked internationally, or being married to another physician who also had limited time for retirement planning added complications to the financial aspects of retirement planning. Being the sole breadwinner also added complexity to retirement plans, as did financial obligations to aging parents, the cost of education for dependents and maintaining current lifestyle expenses. In discussions with more experienced physicians, having a younger partner or spouse who was not interested in retiring, or divorces, were mentioned as impediments to retirement planning.

\section{Rigid institutional structures}

Inflexible practice plans arose as a key barrier to retirement planning. Interviewees suggested that hospital practice plans and administrative leaders favour full retirement transitions. However, most of the focus group participants expressed a desire to retire in a way that allowed them to slowly cut back their hours. There were several participants who suggested that the idea of stopping abruptly was undesirable or even frightening. Having to be on-call at later career stages was described as arduous and an encouragement to abruptly retire.

\section{Professional norms}

Participants described their professional culture as one that favoured work over all other aspects of life, thus constricting their incentives to plan for retirement. Later-career transitions were mentioned as a topic that was not openly discussed despite the consequences that can ensue if a successor is not in place to take over seeing patients and managing one's practice.

Several later-career participants expressed a general sense that transitioning from medicine was not an easy endeavour given the lack of support for retirement planning. Focus group participants and interviewees also repeatedly mentioned that the demands of their career often limited their time to plan for retirement or to develop interests or hobbies, which could be things to look forward to in retirement. Concerns were expressed during the focus groups about the lack of people who could serve as models for successful ways to transition from medicine. Focus group participants found it difficult to reference someone whose transition they would have liked to mimic.

\section{Facilitators to physician retirement planning}

Participants offered a range of suggestions to help physicians embrace retirement planning, which included developing mechanisms that support financial planning resources for physicians at multiple career stages, opportunities and resources for later-career transitions and later-career mentorship, support for intergenerational collaboration and recognition of retirees.

\section{Financial planning resources for physicians at multiple career stages}

Focus group participants and interviewees suggested that the financial aspects of retirement planning should be targeted to physicians at early, mid-, and later-career stages. Several participants had suggestions such as websites that featured spreadsheets with retirement calculators specific to different career stages and linked them with alternative career options. Other suggestions to help facilitate retirement planning included incorporating retirement financial planning (or even sneaking it) into other discussions about career progress, life insurance or powers of attorney.

\section{Opportunities and resources for gradual later career transitions}

Improving flexibility with regard to specific types of work, such as on-call duties, was suggested as a way to improve retirement transition. Participants noted the importance of 
Table 3: Illustrative quotes

Barriers

Poor personal financial management
Facilitators

Financial planning resources for physicians at multiple career stages

"Fiscal advice over the career is one thing that might be very important."

potential options are and how you would want to benefit out of those."

"How much do we need to retire? That's been a problem for me, even though I have a financial advisor who says you can retire, do I believe him?"

"If it's more a long-term plan, it sort-of starts mid-career, and it's just not suddenly as you turn 60 , that someone is starting to ask the question."

"My ideal transition would be facilitated by winning the lottery. We

are a single income family and I will never be able to afford

retirement ... Right now, we live paycheck to paycheck on an

80-hour work week."

\section{Rigid institutional structures}

Opportunities and resources for later career transitions

"It seems to me that ... when you retire, you retire from everything; is that how it works?"

“... My practice plan says that if I don't continue to do call just like everybody else, even though l've done it for 30 years, I can't be part of the practice."

\section{Professional norms}

"So I don't know if I'm going to be able to say 'yes, I will retire' Because when I look at my colleagues ... no one is retiring easily."

"... Many of us, and I'm one of them, have not developed a whole lot of extracurricular interests."

"... People have these amazing careers and you don't know them deeply, and you don't know how much they've done."
"I don't see myself as retired. I see myself transitioning to something else where I feel I also provide a contribution."

"It's not a question of age ... some people are remarkably healthy mentally and physically and can go on forever."

Later career mentorship, support for intergenerational collaboration, and recognition of retirees

"It's knowing who to ask if you're making certain kinds of decisions, and getting the ongoing mentoring, and always revisiting the plan."

"Let's say you have someone who's older, and you have a young faculty person ... There could be job-sharing that goes along. So, in one sense, you have the older person, who's the heir, can bring along the younger person who only wants part-time work at this time." ensuring that physicians were fit for practice, but felt age was a crude and inappropriate indicator of competence. In light of the meaningful contributions that many senior physicians still have to make, it was suggested that offering work-sharing opportunities could create options that would be amenable to later-career physicians in a way that would help facilitate planning. For example, it was suggested that health care organizations could create more flexible work-sharing opportunities involving billing and space allocations between physicians in the early stages of raising a family and later-career physicians.

\section{Later career mentorship, support for intergenerational collaboration and recognition of retirees}

Fostering a workplace culture that supports positive discourse around later-career transitions was suggested as a way to facilitate retirement planning. Mid- and later-career participants expressed their interest in retirement mentorship programs, "transition navigators" or some form of mentorship that included peer support for retirement planning. Creating roles for retirees as paid or unpaid mentors was suggested as a way for retirees to provide guidance while remaining socially engaged within the medical community.

To make retirement planning more palatable, participants suggested having a shared inclusive space for retirees or an environment that provided retirees with a way to be seen and continually engaged with colleagues. Inviting retirees to regularly held special events and providing retirees with specific, paid or voluntary tasks part-time, or flexible roles and continued access to resources such as journal articles and email, were mentioned as factors that could help facilitate physician retirement planning.

\section{Interpretation}

Our findings outline some of the specific barriers to retirement planning, including poor financial planning in light of complex personal lives, institutional frameworks that emphasize fullretirement transitions and perceived professional norms that fail to recognize the value of planning for transitions from medicine. Within medicine, retirement is often euphemistically referred to as transitioning from practice, thus reflecting an aversion to the notion of abruptly terminating work. . $^{2,39,40}$ The integrated findings from the survey and qualitative components of this study further emphasize that physicians prefer to retire gradually and are concerned when faced with the prospect of abruptly stopping work. This supports previous work that highlighted the identity threat physicians may feel during retirement transitions, particularly in a profession that may not allow for the development of outside interests, identities and relationships - a challenge that was also echoed in our results. ${ }^{11}$ 


\section{OPEN}

Addressing these challenges requires a concerted effort, not just by physicians themselves, but by the broader institutions that help shape institutional practices and norms. Retirement incentives, mandatory retirement and physician supply have previously been examined in relation to the challenges associated with retirement..$^{24,41-44}$ This study contributes by offering a set of specific facilitators to retirement planning. Education and financial planning resources throughout career stages could provide physicians with tools to approach their personal planning. Additionally, the perception that institutional structures may not allow for gradual retirement suggested the need for enhanced flexibility in institutional retirement plans. Prior work in the United States has highlighted the need to create flexible, phased or part-time retirement as viable options..$^{25}$ This is supported by previous research that suggests institutional arrangements that allow physicians to work part-time can lead to later retirement. ${ }^{26,45}$

Some aspects of retirement planning that affect pensions, such as challenges related to sharing billing numbers in the case of joint practices, were not raised as key concerns by participants in this study despite their relevance as evidenced by a systematic review of physician retirement planning. ${ }^{46}$ This may be because some academic-affiliated physicians have pension plans not available to community physicians who may perceive practice costs as a disincentive to continue working later in their career.

Finally, mentorship has been a focus of much study for earlier career development in medicine, ${ }^{47,48}$ and findings from this study point to the need to examine mentorship for later-career physicians. This study also supports shifting away from agebased retirement norms and instead focusing on how medical institutions can create mutually respectful retirement transitions by promoting planning activities at various career stages.

\section{Limitations}

Although this study benefited from its use of multiple methods including focus groups, an online survey and interviews from participants at a large academic centre with numerous affiliated hospitals, a number of limitations should be noted. The survey presents the risk of participant bias if, for example, respondents who felt strongly about certain retirement issues were more likely to participate. Another limitation of this study is the involvement of participants from a large urban academic centre. Although there were benefits to studying an academic centre, this does restrict the generalizability of our results, because academic centres tend to be concentrated in more urban areas and may represent only a small subsection of the practice population of more community-based specialties, such as family medicine. Rural areas, or areas that are underserved, may face particular challenges in approaching retirement planning that are not addressed here. ${ }^{49}$ In addition, participants were all fully employed physicians; although they could contribute their perspectives on retirement planning, none of them were actually retired. Therefore, we can only draw conclusions on the perceived barriers and facilitators to retirement planning, but not to retirement itself.

\section{Conclusion}

This study examined perceived barriers to and facilitators of retirement planning among a sample of academic physicians from a range of medical specialties, at different career stages. Findings suggest that having flexible options for retiring and creating a culture that supports discussions about later-career transitions and that continues to honour and involve retirees, can help enhance physicians' retirement planning. The results of this study suggest that medical institutions can facilitate retirement planning through efforts to promote tools to guide financial planning and mentorship programs at various career stages. This field would benefit from future studies examining potential sex differences in retirement planning in addition to differences based on region and practice setting. Future research should examine later-career mentorship programs and work-share models that aim to enhance retirement transitions.

\section{References}

1. Fralick M, Flegel K. Physician burnout: Who will protect us from ourselves? CMA7 2014;186:731.

2. Pong R. Putting away the stethoscope for good? Toward a new perspective on physician retirement. Ottawa: Canadian Institute for Health Information; 2011:1-83.

3. Schellenberg G, Ostrovsky Y. Canadian social trends: 2007 General Social Survey Report: the retirement plans and expectations of older worker. Ottawa: Statistics Canada; 2008. Available: www.statcan.gc.ca/pub/11-008-x/2008002/ article/10666-eng.htm (accessed 2016 Aug. 5).

4. Martin S. Freedom 55 closer to age 65 for physicians. CMA7 2000;163:1499.

5. Miller R. December financial checkup for physicians. 7 Med Pract Manage 2014;30:164-5.

6. Anthony R, Ezat W, Junid S, et al. Financial management attitude and practice among the medical practitioners in public and private medical service in Malaysia. Int 7 Business Manage 2011;6:105-13.

7. Collier R. Learning to retire gracefully. CMA7 2011;183:E1282.

8. Schlossberg N. Revitalizing retirement. Washington (DC): American Psychological Association; 2009.

9. Levis JB, Markle D. Retirement planning for physicians. Adv Anesth 2009; 27:95-110.

10. Dhaliwal G, Chou C. A brief educational intervention in personal finance for medical residents. 7 Gen Intern Med 2007;22:374-7.

11. Onyura B, Bohnen J, Wasylenki D, et al. Reimagining the self at late-career transitions. Acad Med 2015;90:794-801.

12. Doyle $\mathrm{S}$. Is there life after medicine? CMA7 2011;183:E72

13. Walker Keegan D. Physician transition plans: planning for physician slowdown. 7 Med Pract Manage 2008;23:363-6.

14. Pong RW, Lemire F, Tepper J. Physician retirement in Canada: what is known and what needs to be done. Proceedings of the 10th International Medical Workforce Conference Vancouver: Medical Workforce Conference; 2007 Mar. 20-24; Vancouver. Available: http://rcpsc.medical.org/publicpolicy/imwc/10_ ret\&retCAN.pdf (accessed 2016 Aug. 5).

15. Chan B, Anderson GM, Theriault M. Patterns of practice among older physicians in Ontario. CMA7 1998;159:1101-6.

16. Wijeratne $C$, Peisah C. Accepting the challenges of ageing and retirement in ourselves: The need for psychiatrists to adopt a consensus approach. Aust NZ 7 Psychiatry 2013;47:425-30.

17. Peisah C, Wilhelm K. Physician don't heal thyself: a descriptive study of impaired older doctors. Int Psychogeriatr 2007;19:974-84.

18. Day GS, Carette S, Tang-Wai DF. Cognitive decline in an older physician. CMA7 2015;187:750-4.

19. McKenna M. Aging gracefully?: Patient safety advocates call for ongoing skills assessments for older physicians. Ann Emerg Med 2011;58:A15-7.

20. Blasier RB. The problem of the aging surgeon: when surgeon age becomes a surgical risk factor. Clin Orthop Relat Res 2009;467:402-11.

21. Noone J, Stephens C, Alpass F. Preretirement planning and well-being in later life: A prospective study. Res Aging 2009;31:295-317.

22. Wong J, Earl J. Towards an integrated model of individual, psychosocial, and organizational predictors of retirement adjustment. 7 Vocat Bebav 2009; 75:1-13.

23. Kim JE, Moe P. Retirement transitions, gender, and psychological well-being: A life-course ecological model. 7 Gerontol B Psychol Sci Soc Sci 2002;57:P212-22.

24. Trent B. Mandatory retirement: should older MDs be forced to retire to make way for the new? CMA7 1993;149:1696-9.

25. Moss AJ, Greenberg H, Dwyer EM, et al. Senior academic physicians and retirement considerations. Prog Cardiovasc Dis 2013;55:611-5. 
26. Merline AC, Cull WL, Mulvey HJ, et al. Patterns of work and retirement among pediatricians aged $\geq 50$ years. Pediatrics 2010;125:158-64.

27. Pololi LH, Krupat E, Civian JT, et al. Why are a quarter of faculty considering leaving academic medicine? A study of their perceptions of institutional culture and intentions to leave at 26 representative US medical schools. Acad Med 2012;87:859-69.

28. Silver MP, Pang NC, Williams SA. Why give up something that works so well? Retirement expectations among academic physicians. Educational Gerontol 2015;41:333-47.

29. Strong EA, De Castro R, Sambuco D, et al. Work-life balance in academic medicine: narratives of physician-researchers and their mentors. 7 Gen Intern Med 2013;28:1596-603.

30. Creswell JW, Clark VLP, editors. Designing and conducting mixed methods research. 2nd ed. Thousand Oaks (CA): Sage; 2011.

31. Ilic D, Hart $W$, Fiddes $\mathrm{P}$, et al. Adopting a blended learning approach to teaching evidence based medicine: a mixed methods study. BMC Med Educ 2013;13:169.

32. Gilbert A, Francischetto EO, Blazeby J, et al. Choice of a patient-reported outcome measure for patients with anal cancer for use in cancer clinical trials and routine clinical practice: a mixed methods approach. Lancet 2015; 385(Suppl 1):S38.

33. Rogers A, Harris T, Victor C, et al. Which older people decline participation in a primary care trial of physical activity and why: insights from a mixed methods approach. BMC Geriatr 2014;14:46.

34. Tashakkori A, Teddlie C. Handbook of mixed methods in social and behavioral research. Thousand Oaks (CA): Sage; 2003.

35. Braun V, Clarke V. Using thematic analysis in psychology. Qual Res Psychol 2006;3:77-101.

36. Fereday J. Demonstrating rigor using thematic analysis: a hybrid approach of inductive and deductive coding and theme development. Int 7 Qual Methods 2006;5:80-92.

37. Creswell J, Miller D. Determining validity in qualitative inquiry. Theory Pract 2000;39:124-30.

38. Sandelowski M. Rigor or rigor mortis. ANS Adv Nurs Sci 1993;16:1-8.

39. Collier R. Diagnosing the aging physician. CMA7 2008;178:1121-3.

40. Goldberg R, Thomas H, Penner L. Issues of concern to emergency physicians in pre-retirement years: a survey. 7 Emerg Med 2011;40:706-13.

41. Golden BR, Hannam R, Hyatt D. Managing the supply of physicians' services through intelligent incentives. CMA72012;184:E77-80

42. Dauphinee WD. Medical workforce policy making in Canada: Are we creating more problems for the future? Clin Invest Med 1996;19:286-91.

43. Fletcher SL, Schofield DJ. The impact of generational change and retiremen on psychiatry to 2025. BMC Health Serv Res 2007;7:141.

44. Gillies JH, Ross LC. Physician retirement: a case for concern in Canadian hospitals. Can Med Assoc f 1984;131:297-9.

45. Nusbaum NJ. Commentary: physician retirement and physician shortages. 7 Community Health 2009;34:353-6.

46. Silver MP, Hamilton AD, Biswas A, et al. A systematic review of physician retirement planning. Hum Resour Health 2016;14:67.

47. Sambunjak D, Straus SE, Marusic A. Mentoring in academic medicine: a systematic review. FAMA 2006;296:1103-15.

48. Straus SE, Chatur F, Taylor M. Issues in the mentor-mentee relationship in academic medicine: a qualitative study. Acad Med 2009;84:135-9.

49. Hansen V, Pit SW, Honeyman P, et al. Prolonging a sustainable working life among older rural GPS: solutions from the horse's mouth. Rural Remote Health 2013;13:2369.

Affiliation: Institute of Health Policy, Management and Evaluation (Pannor Silver, Easty); Department of Health Studies/Anthropology (Pannor Silver), University of Toronto, Toronto, Ont.

Contributors: Michelle Pannor Silver conceived the study and led the overall study design. Michelle Pannor Silver coordinated the focus groups and conducted all interviews. Michelle Pannor Silver and Laura Easty worked together to analyze the findings and draft the manuscript. Both authors approved the final version to be published and agreed to acts as guarantors of the results.

Funding: Funding support for the study was provided by the University of Toronto Department of Medicine.

Supplemental information: For reviewer comments and the original submission of this manuscript, please see www.cmajopen.ca/content/5/1/ E123/suppl/DC1 